\title{
Venomous Terrestrial Snakes in the Tropics of Malaysia: Review
}

\author{
Iman D. Johan Arief ${ }^{1,2 *}$, Xin Y. Er ${ }^{1,2}$, Rafiq Shajahan1, Faiz Johan Arief ${ }^{1}$, Naganathan Pillai1 \\ ${ }^{1}$ Monash University Malaysia, Selangor, Malaysia \\ ${ }^{2}$ Royal Darwin Hospital, Darwin, Australia \\ Email: ^imandanie191@gmail.com
}

How to cite this paper: Arief, I.D.J., Er, X.Y., Shajahan, R., Arief, F.J. and Pillai, N. (2021) Venomous Terrestrial Snakes in the Tropics of Malaysia: Review. Open Access Library Journal, 8: e7231.

https://doi.org/10.4236/oalib.1107231

Received: February 8, 2021

Accepted: March 21, 2021

Published: March 24, 2021

Copyright $\odot 2021$ by author(s) and Open Access Library Inc.

This work is licensed under the Creative Commons Attribution International License (CC BY 4.0).

http://creativecommons.org/licenses/by/4.0/

(c) (i) Open Access

\begin{abstract}
The tropics of Malaysia have plenty to offer when it comes to its biodiversity. In the animal kingdom, snakes play a crucial role and form a large component in the ecosystem, with human envenomation being a fairly common problem. From 1965 to 1971, there were 5203 reported cases of venomous snake bites throughout Malaysia, 18 of which being fatal cases. Symptomatology varies, ranging from mild local symptoms on one end of the spectrum to life threatening systemic manifestation on the other end. Quick identification of species and application of basic first aid management is crucial in preventing further complications, whilst awaiting further definitive management if necessary. Thus, a well-established management plan for selecting cases based on recognition of species would ultimately influence the prognosis of victims.
\end{abstract}

\section{Subject Areas}

Environmental Sciences, Toxicology, Zoology

\section{Keywords}

Venom, Toxins, Snakes, Malaysia, Anti-Venom

\section{Introduction}

Our planet, Earth, has been blessed by a biodiversity of unparalleled proportions. It is truly remarkable and humbling. However, within this level of variance in life, toxic and poisonous creatures are present in equal numerical richness [1]. These organisms have been capturing our fascination since early recorded history and we have learnt to co-exist peacefully over a millennia [2]. In spite of this, it is important to learn about these organisms as they secrete bioactive mo- 
lecules and poisons that cause various detriments to the body [3].

It is crucial to understand the basic terminologies that will be used throughout the article as they are very well not interchangeable. Firstly, toxins are chemical compounds that can damage other organisms at a cellular level [1]. Venoms and poisons are both forms of toxins which can be utilized by certain organisms, either as a means of defence or offense. They however differ in terms of delivery and its origins. Poisons are toxins that are passively exposed on a particular organism, for example, the body of a poison dart frog which can be highly poisonous [2]. This is in contrast with venom, which is essentially toxins that are actively injected or introduced into a victim via a set of specialized apparatus such as that of a snake [2] [3] [4]. Although the majority of these apparatus are spindle or needle-like in nature, some species may transfer their venom through forward facing holes at the tips of fangs (such as in the Naja Sp. of African spitting cobras) [1] [5]. To simply put it: If you bite something and die, it is a poison or a poisonous organism. However, if something bites you and you die, it is a venomous organism. Hence, these are not interchangeable terminologies and it is very important especially in various fields that apply the basic of toxicology and to specifically distinguish the method of toxin delivery utilised by the relevant venomous terrestrial snakes mentioned in this article.

\section{Venomous Snakes of Malaysia}

Malaysia is a country of the tropics, located at the equator, well known for its warmth and humidity all year round. Many species of venomous snakes form part of the diverse ecosystem found here in Malaysia. Snake bites are very common especially in rural areas. Envenomation of certain species can result in serious medical problems. Clinical manifestations can often vary, depending on the nature of the venom. This may range from having disfiguring scars and deformities to serious neurological sequelae with high rates of fatality.

\subsection{Medically Significant Species of Snakes of Malaysia}

Generally, the venomous terrestrial species that account for hospitalizations can be categorized into two broad families - Elapidae and Viperidae (Table 1) [6].

\subsection{Snake Bite Cases in Peninsular Malaysia}

Table 2 shows the statistics on snake bites cases in Peninsular Malaysia. Figure 1 and Figure 2 show the top and lateral view of the head of a cobra and a Malayan pit viper.

\subsection{Elapidae}

Cobras, coral snakes, sea kraits and sea snakes are all found within this family. This family is further subdivided into 5 subfamilies: Elapinae, Hydrophiinae, Laticaudinae, Ephalophiini and Hydrophiini. The distinguishing feature that set members of this family apart from non-venomous species are the short fangs 
Table 1. Venomous terrestrial species of snakes in Malaysia, divided into 2 families, the elapidae which include cobras and kraits and viperidae which include vipers and pit vipers.

\begin{tabular}{|c|c|c|c|c|}
\hline Family & Subfamily & Genus & Scientific Name & Common Name \\
\hline \multirow{7}{*}{ Elapidae } & \multirow{7}{*}{ Elapinae } & Naja & Naja naja sputatrix & Malayan cobra \\
\hline & & & Naja naja kaouthia & Monocled cobra \\
\hline & & & Naja sumatrana & Equatorial spitting cobra \\
\hline & & Bungarus & Bungarus candidus & \\
\hline & & & Bungarus fasciatus & \\
\hline & & & Bungarus flaviceps & \\
\hline & & Ophiophagus & Ophiophagus hannah & King cobra \\
\hline \multirow{7}{*}{ Viperidae } & \multirow{7}{*}{ Crotalinae } & \multirow{3}{*}{ Calloselasma } & Calloselasma rhodostoma & Malayan pit viper \\
\hline & & & Trimeresurus wagleri & Wagler's pit viper \\
\hline & & & Trimeresurus purpureomaculatus & Shore pit viper \\
\hline & & \multirow{4}{*}{ Trimeresurus } & Trimeresurus sumatranus & Sumatran pit viper \\
\hline & & & Trimeresurus albolabris & White-lipped tree viper \\
\hline & & & Trimeresurus popeiorum & Pope's pit viper \\
\hline & & & Trimeresurus monticola & Mountain pit viper \\
\hline
\end{tabular}

Table 2. Statistics on species of snake bites throughout Malaysia from 1965-1971 [7].

\begin{tabular}{ccc}
\hline Snake Species & Total Cases & Fatal Cases \\
\hline Malayan pit viper (Calloselasma rhodostoma) & 1136 & 4 \\
Sea snakes & 158 & 5 \\
Asian common cobra (Naja naja) & 112 & 3 \\
Asian lance-headed viper (Trimeresurus) & 25 & 0 \\
King cobra (Ophiophagus hannah) & 6 & 0 \\
Krait (Bungarus) & 1 & 0 \\
Unidentified & 3765 & 0 \\
Nonpoisonous & 184 & \\
\hline
\end{tabular}
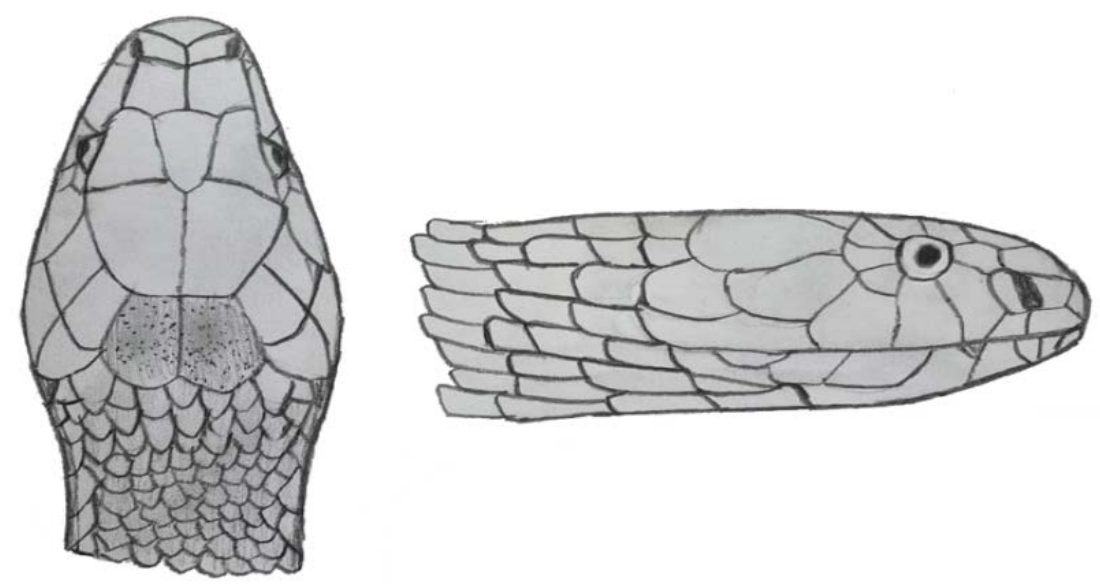

Figure 1. Diagram shows the head of a cobra (top and lateral view). Hand drawn by Faiz Johan Arief. 


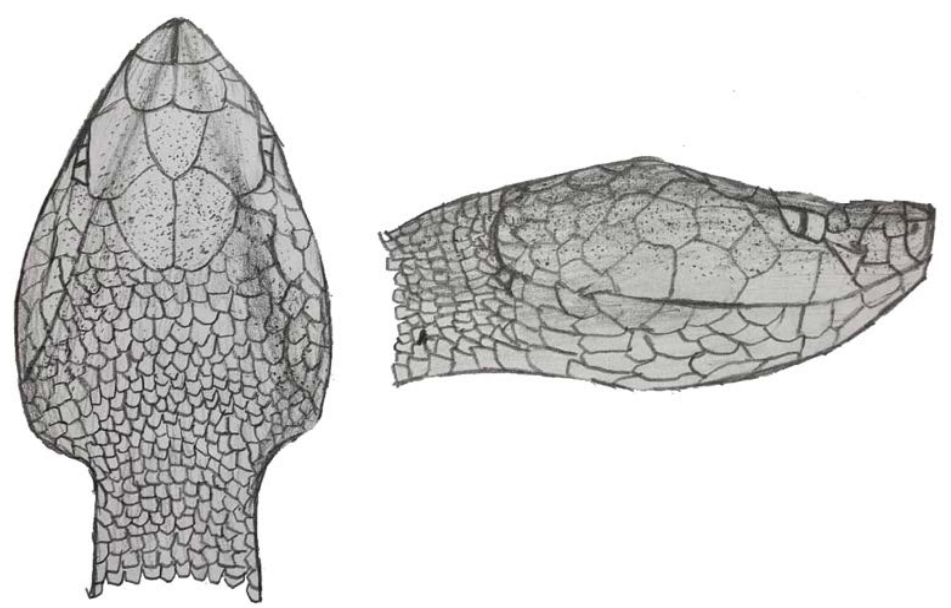

Figure 2. Diagram shows the top and lateral view of the head of a Malayan pit viper. In general, the head of a viper is shaped more triangularly with a pointy snout as opposed to its elapid counterparts. Hand Drawn by Faiz Johan Arief.

placed anteriorly on the front part of the maxilla [7]. The head is usually continuous or only slightly differentiated from the rest of the body. The subfamily Elapinae would be discussed here whereas the sea snakes would be discussed in a later chapter.

In Malaysia, cobra bites while uncommon, can result in mortality. These species are usually not aggressive and tend to stay clear of human contact if left alone. However, if provoked, cobras may flare out their "hood", often as a means of warning to predators or humans to keep their distance [7].

Among the 2 subspecies of Asian cobras, the (Naja naja) is responsible for venomous snake bites in Malaysia. The Malayan cobra (Naja naja sputatrix) is usually a brownish black snake with white makings on its throat [7]. They possess post-synaptic neurotoxins, known as sputa-neurotoxin 1 and sputa-neurotoxin 2 [7]. Other biochemical composition of the venom includes polypeptide cardiotoxins and phospholipase A2 [7] [8].

The $2^{\text {nd }}$ subspecies of Asian cobras, Monocled cobra (Naja naja kaouthia) (Figure 3), are more commonly found in the northern states of Malaysia. A distinct white ring-like circle is usually visible at the back of its hood [7]. Their venom constituents include postsynaptic neurotoxins, cardiotoxins and phospholipase A2. Despite originating from the same species and having the same types of venom, the venom composition Naja naja sputatrix cobra differs strikingly with Naja naja kaouthia, as it has a higher content of neurotoxins and lower cardiotoxins composition. Thus, this may explain why antivenoms towards the Naja naja kaouthia are ineffective in cases of snake bites from the Naja naja sputatrix [7].

Another species of cobra that is widely present over most of Peninsula Malaysia is the equatorial spitting cobra (Naja sumatrana) (Figure 4). The cobra comes in two different colours, depending on its phase; notably the black phase and golden phase. It is infamous for its capability of "spitting" its venom into the 


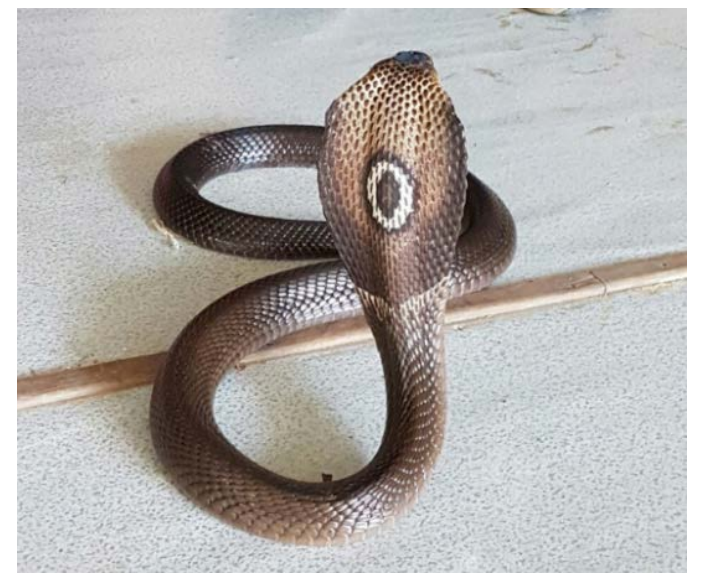

Figure 3. Monocled cobra (Naja kaouthia), with its signature O-shaped or monocellate pattern at the back of its hood, is the easiest distinguishing feature when compared to other cobra species.

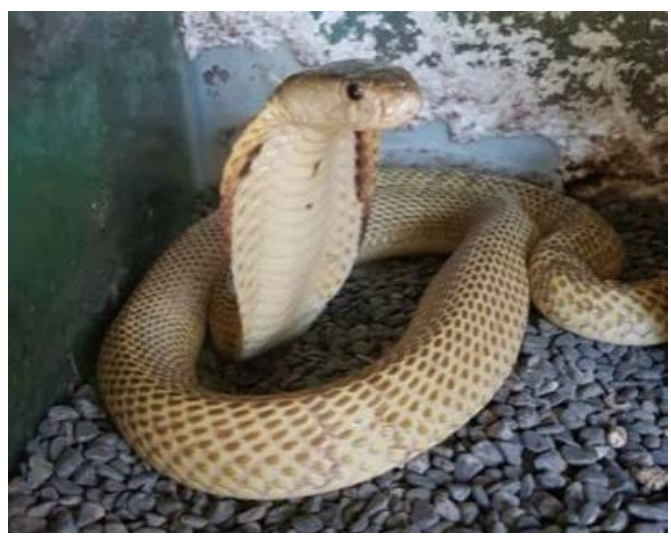

Figure 4. Golden phase of the equatorial spitting cobra (Naja sumatrana). It is capable of spraying its venom, in a jet-like propulsion with pin-point accuracy aiming for the eyes.

eyes of an aggressor, resulting in a clinical entity known as cobra-spit ophthalmia [7]. Early symptoms include red, painful and watery eyes, with photophobia, blurring of vision and temporary blindness ensuing later. Corneal ulceration and scarring are known complications of the venom if left untreated [7] [9].

The King cobra (Ophiophagus hannah) (Figure 5 and Figure 6), is renowned for its enormous size. It carries an impressive reputation of being the world's largest venomous snake, with specimens reaching up to 5.4 meters in length [7] [8]. Another distinguishing feature of a king cobra is the occipital shields located on top of its head [8]. However, bites from king cobras are rare in Malaysia. Nevertheless, bites need to be treated acutely and competently, as the quantity of venom injected can be of a large amount [7]. The venom of Ophiophagus hannah markedly differs compared to the Naja genus, in part due to a much higher enzyme constituent, leading to more inflammatory reactions [7] [10]. Snake venom metalloproteinases (SVMPs) are present in extensive amount, with the haemorrhagic protease hannah toxin being one of the major component in the venom [10]. Its pro-inflammatory activity together with proteolytic action 


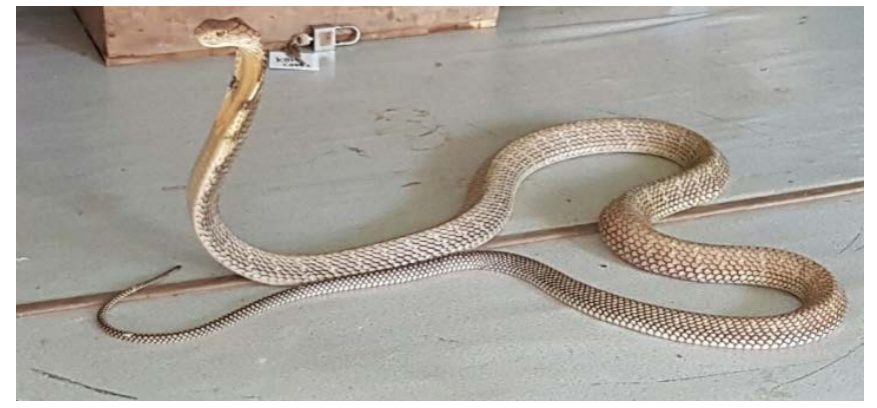

Figure 5. The king cobra (Ophiophagus hannah) is renowned for its enormous size, gaining the title of world's largest venomous snake, with adult specimens capable of growing up to 5.4 meters in length.

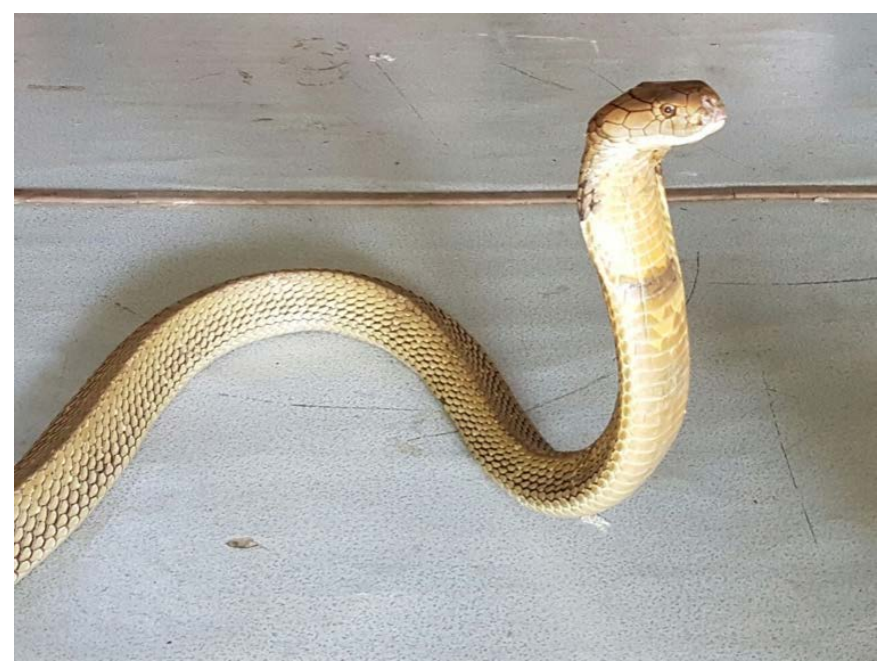

Figure 6. For what they lack in quality, they simply make it up in quantity. The king is capable of injecting up to $500 \mathrm{mg}$ of venom into its victim resulting in serious complications.

may result in cytotoxicity, leading to local tissue necrosis [8] [12]. Other toxins found include various postsynaptic neurotoxins which may account for the often observed complications of systemic neurotoxicity present in many envenomation [7].

Their venom (median lethal dose, $\mathrm{LD}_{50}$ of 1.2) is not as potent as compared to that of Naja kaouthia or Naja sputatrix (median lethal dose, $\mathrm{LD}_{50}$ of 0.4 and 0.8 respectively) [8] [12]. However, the amount of venom injected can be substantial (up to 350 to $500 \mathrm{mg}$ ), which rapidly leads to systemic neurotoxicity, requiring a large dose of antivenom to reverse its effects [7] [10].

Generally, the bites of most elapids result in clinical neurotoxicity. The post-synaptic neurotoxins act as a competitive antagonist at the nicotinic acetylcholine receptors, thus producing a depolarising neuromuscular blockade [7]. Clinical manifestation therefore, includes muscular paresis and flaccid paralysis, drowsiness, dysphagia, ptosis with subsequent respiratory arrest [7] [8].

Besides that, the Naja genus also possesses cardiotoxins which may cause haemolysis due to ion pores formation in the lipid bilayer membranes [13]. Also, 
cardiotoxins are capable of inducing an influx of calcium $\left(\mathrm{Ca}^{2+}\right)$ into the cells, leading to muscle depolarization causing muscle contractures [8] [11].

Another important venom constituent in some elapid species are phospholipase A2. It has been thought that the synergistic effect of cardiotoxins and phospholipase A2 results in local tissue necrosis and inflammation [7].

\subsection{Viperidae}

There are 3 subfamilies within this family of vipers; the Fea's viper (Azemiopinae), pit vipers (Crotalinae) and pitless vipers or true vipers, (Viperinae) [7]. The subfamily pit vipers, Crotalinae, is responsible for majority of the snake bites in Malaysia. As the name suggest, members of this subfamily of snakes are unique as they have sensory pits located between the eye and the nostrils, which act as an external opening to a pair of thermo-sensitive organs. Thus, they are able to hunt efficiently in complete darkness by detecting the presence of heat within warm-blooded animals [12]. Despite the high reports of snake bites due to pit vipers in Malaysia, most envenomation does not result in fatality [12]. Nevertheless, the bites can be excruciatingly painful with local blistering, tissue necrosis and haemorrhage [7].

Their heads are usually broad and distinct as opposed to the elapidae family. Besides that, they usually have a relatively short and stubby appearance. It is believed that vipers have the most sophisticated venom inoculating apparatus when compared to other snakes. Their large fangs are able to penetrate deeply into their prey. Also, when not in use, the fangs are orientated in a parallel manner against the upper jaw with its mouth closed. The snake however, is able to lift and move its fangs 90 degrees forward to attack its prey [7] [12].

The infamous Malayan pit viper (Calloselasma rhodostoma) (Figure 7) is

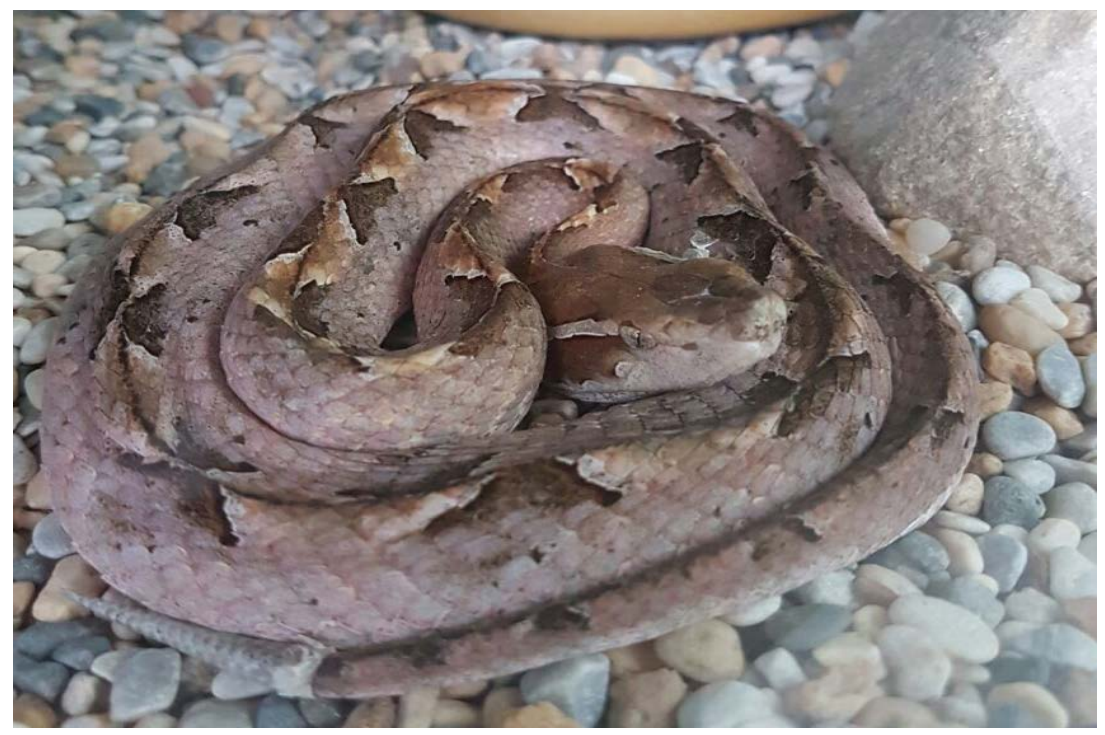

Figure 7. The Malayan pit viper (Calloselasma rhodostoma) is an extremely important venomous snake in Malaysia, responsible for the majority of snake bite cases in Malaysia due to its highly camouflage body and its frequent encounters with humans. 
known to be frequently implicated in many human snake bite reports. The bites are usually confined to the northern states, in Penang, Kedah and Perlis. Despite its limited distribution geographically, it is still responsible for the most number of snake bite cases in Peninsular Malaysia [7]. Its effective camouflage, sluggish character and the fact that it spends a lot of time on the ground could contribute to the substantial number of envenomations [7] [12]. The body is triangular in cross section with a flat triangular head that is very distinct [12]. The head is generally rusty red in colour and the body has a reddish-brown coloration with blotches of darker shade of semi triangles extending from the midline, alternatingly from each side [12].

The venom is comprised of various different enzymes and proteins, with a lower potency and lethality compared to the venoms of most elapids. An important enzyme present in these venoms called Ancrod, is a thrombin-like enzyme. It serves to form clots that are not crossed-linked. Human plasmin in turn will then rapidly lyse these clots. Hence, in vitro, Ancrod is a pro-coagulant but when it is exposed to victims, overt haemorrhage results in part due to the ensuing low levels of fibrinogen [7] [13].

Besides that, the venom also contains haemorrhagins (a type of metalloproteinase). It has been postulated that haemorrhagins result in the destruction of collagenous basement membrane and the weakening of the endothelial wall of blood vessels, making them more susceptible to rupture and haemorrhage [7]. The chief Malayan pit viper haemorrhagin is rhodostoxin, known for its potent haemorrhagic activity and oedema-inducing capability [7].

Another important component of the venom is platelet aggregation inducers known as aggretin (rhodocytin) and platelet aggregation inhibitors known as disintegrins [13]. While aggretin is thought to result in a thrombocytopenic syndrome, disintegrins plays a major role in preventing platelet aggregation by disrupting the binding of fibrinogen to integrin of platelets. These venom components synergistically exacerbate the haemorrhagic activity further [7].

Other constituents of the venom are as follows:

1) Fibrinolytic factors which contributes to defibrination syndrome,

2) Oedema-forming factors,

3) L-amino acid oxidase with platelet aggregation impairment activity, and

4) Hyaluronidase, known as the "spreading factor" that helps with venom absorption in the host or prey [13].

Local effects of envenomation include gross oedema with extensive tissue necrosis [7]. Systemic manifestation of Malayan pit viper envenomation includes systemic haemorrhage with defibrination and thrombocytopenia [7]. Symptoms such as gingivial bleeding may arise, followed by epistaxis, haemoptysis, petechiae, purpura and ecchymoses [7]. Severe complications following the envenomation often include intracranial bleed and retroperitoneal haemorrhage. Overt bleeding may eventually predispose the patient to shock secondary to hypovolemia [7].

The genus Trimeresurus (Asian lance-headed pit vipers) are pit vipers with 
scales on top of their head that are rather small and irregularly placed. This is in contrast with the Malayan pit viper (Calloselasma rhodostoma) discussed earlier, in which their large scales on the head are systematically in place. There are a few species within this genus that are responsible for a minority of cases of snake bites in Malaysia [7]. Table 3 shows the summary of pit vipers.

Generally, the bites of members of this genus rarely result in serious envenomation, with localized oedema and accompanying pain. The potency of the haemorrhagic and thrombin-like activity varies depending on the species, resulting in thrombocytopenic syndrome and haemorrhage. Bites are rare and usually do not result in mortality, though it can be excruciatingly painful [7].

Wagler's pit viper (Trimeresurus wagleri) (Figure 8) is among the commonest species of pit vipers found in Malaysia. Due to its highly differentiated morphological characteristics, it has now been categorized in a different subgenus known as Tropidolaemus [7]. Juvenile species are bright green with half-red half-white small spots distributed along its body [12]. The bright green coloration tends to fade in adult specimens, resulting in a variation of colours and patterns, with the typical phenotype having a black base with numerous green spots scattered accompanied with cross-bars that are green above and yellow by the sides [12]. Its habitat includes lowland areas in forests and it is an arboreal species, spending a lot of time climbing the low bushes [7]. Its venom, unlike other pit vipers, only exhibits weak thrombin-like activity with no haemorrhagic or anti-coagulant action. 2 major lethal toxins isolated from this species include the

Table 3. Summary of pit vipers.

\begin{tabular}{|c|c|c|}
\hline $\begin{array}{l}\text { Common Name } \\
\text { (Scientific Name) }\end{array}$ & Physical Description & Venom \\
\hline $\begin{array}{l}\text { Wagler's pit viper } \\
\text { (Trimeresurus wagleri) }\end{array}$ & $\begin{array}{l}\text { Juvenile members of the species are bright green with half-red half-white } \\
\text { small spots distributed along its body [12]. Adult specimen typically has a } \\
\text { black base with numerous green spots scattered accompanied with } \\
\text { cross-bars that are green above and yellow by the sides. }\end{array}$ & $\begin{array}{l}\text { Weak thrombin-like activity with no } \\
\text { hemorrhagic activity. } \\
2 \text { major lethal toxins are wagleri toxin } 1 \\
\text { and } 2[14] \text {. }\end{array}$ \\
\hline $\begin{array}{l}\text { Shore pit viper } \\
\text { (Trimeresurus } \\
\text { purpureomaculatus) }\end{array}$ & $\begin{array}{l}\text { Uniformly brownish-purple though variations include brownish-grey or } \\
\text { olive [7] [12]. Typically found near mangrove swamps and never far off } \\
\text { from the sea. }\end{array}$ & $\begin{array}{l}\text { Potent hemorrhagic activity } \\
\text { (maculatoxin) and modest } \\
\text { thrombin-like activity (purpurase) [14]. }\end{array}$ \\
\hline $\begin{array}{l}\text { Sumatran pit viper } \\
\text { (Trimeresurus sumatranus) }\end{array}$ & $\begin{array}{l}\text { Green with black bands at every } 4 \text { to } 5 \text { scales along its body [12]. Grows } \\
\text { well over one meter in length making it one of the larger pit vipers. }\end{array}$ & $\begin{array}{l}\text { Strong hemorrhagic activity and weak } \\
\text { thrombin-like effects [14]. }\end{array}$ \\
\hline $\begin{array}{l}\text { Pope's tree viper } \\
\text { ( Trimeresurus popeorum) }\end{array}$ & $\begin{array}{l}\text { It is green on the upper body, and pale green or yellow on the lower body. } \\
\text { Separating these } 2 \text { shades of green is a bright bicolored orange and white } \\
\text { lining extending throughout the length of the snake. Its tail is generally red } \\
\text { especially towards the tip [12]. }\end{array}$ & Not available. \\
\hline $\begin{array}{l}\text { White lipped pit viper } \\
\text { (Trimeresurus albolabris) }\end{array}$ & $\begin{array}{l}\text { As the name suggests the upper part of the lips are white, though they can } \\
\text { come in other colors such as pale green or yellow [7]. The body is green in } \\
\text { color. }\end{array}$ & Not Available. \\
\hline $\begin{array}{l}\text { Mountain pit viper } \\
\text { (Trimeresurus monticola) }\end{array}$ & $\begin{array}{l}\text { Brown with black square-like spots on the back, arising from the midline } \\
\text { in an alternating or conjoined fashion. There is also a lighter brownish } \\
\text { yellow coloration extending behind the eyes with large supraocular scales. } \\
\text { Usually found in places of high altitudes [12]. }\end{array}$ & Not Available \\
\hline
\end{tabular}




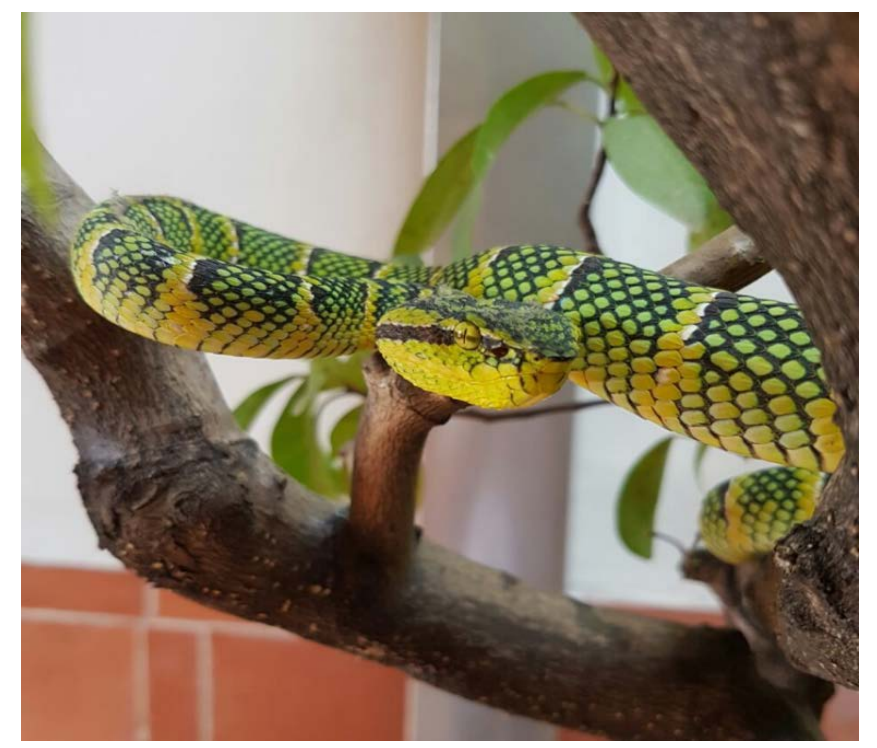

Figure 8. The Wagler's or temple pit vipers (Trimeresurus wagleri) are common with juvenile specimens having a uniformly green body with small spots of red and white throughout the body. As it grows, it attains a greenish and yellowish coloration with bands throughout.

wagleri toxins 1 and 2 [14]. Nevertheless, the bite can result in intense pain accompanied by local oedema.

The Shore pit viper (Trimeresurus purpureomaculatus) (Figure 9), as its name implies, is found near sea shores, particularly along the mangrove swamps. It is usually uniformly brownish-purple though variations include brownish-grey or olive [7] [12]. Its venom has a potent haemorrhagic activity (due to a toxin known as maculatoxin) with modest thrombin-like enzyme (known as purpurase). In addition, unlike most of the other members within the family Crotalidae, the venom of the shore pit viper also demonstrates acetylcholinesterase activity, though its toxic action role is still unclear [7].

The Sumatran pit viper (Trimeresurus sumatranus) (Figure 10) is a highly arboreal species. Found in the forest, it is green with black bands at every 4 to 5 scales along its body. It is capable of growing well over one meter in length, making it one of the larger species of pit vipers [7]. Its venom contains strong haemorrhagic activity but feeble thrombin-like effects [14].

Pope's tree viper (Trimeresurus popeorum) (Figure 11) inhabits the forests on hilly areas (1000 meters above sea level) with a wide geographical distribution [12]. It is green on the upper body, and pale green or yellow on the lower body. Separating these 2 green colour tones is a bright bicolored orange and white lining extending throughout the entire length of the snake. Its tail is generally red especially towards the tip [12]. Pope's tree viper envenomation results in serious haemorrhage and thrombocytopenic syndrome, in accordance to the strong thrombin-like and haemorrhagic activity of the venom [8].

White lipped pit vipers (Trimeresurus albolabris) (Figure 12), inhabits the forest of East Malaysia [7]. As the name suggests the upper part of the lips are 


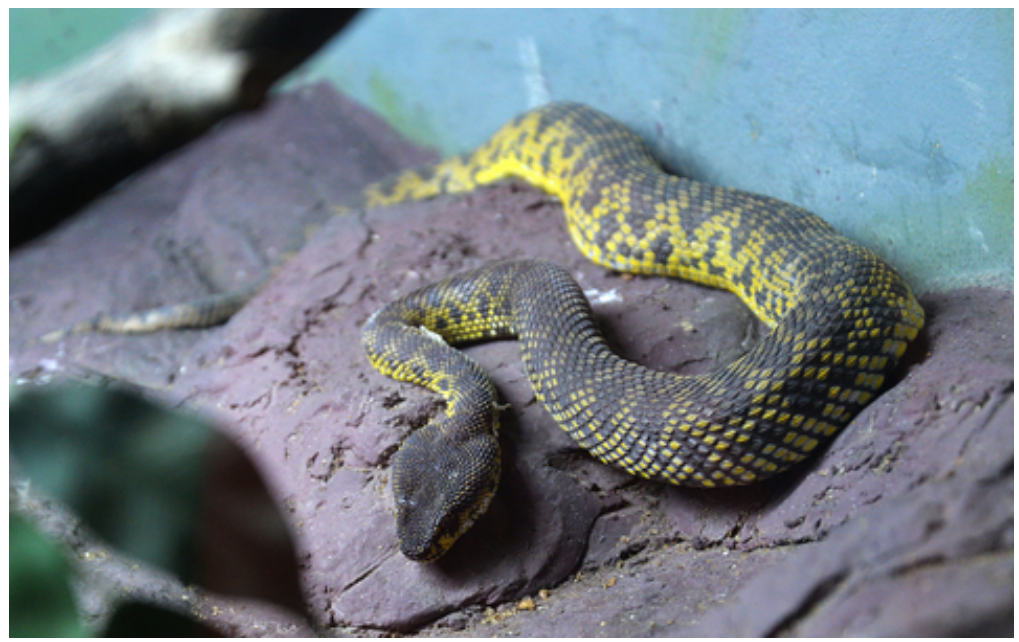

Figure 9. The shore pit viper or otherwise known as the mangrove pit viper (Trimeresurus purpureomaculatus) is typically found in coastline near mangrove swamps.

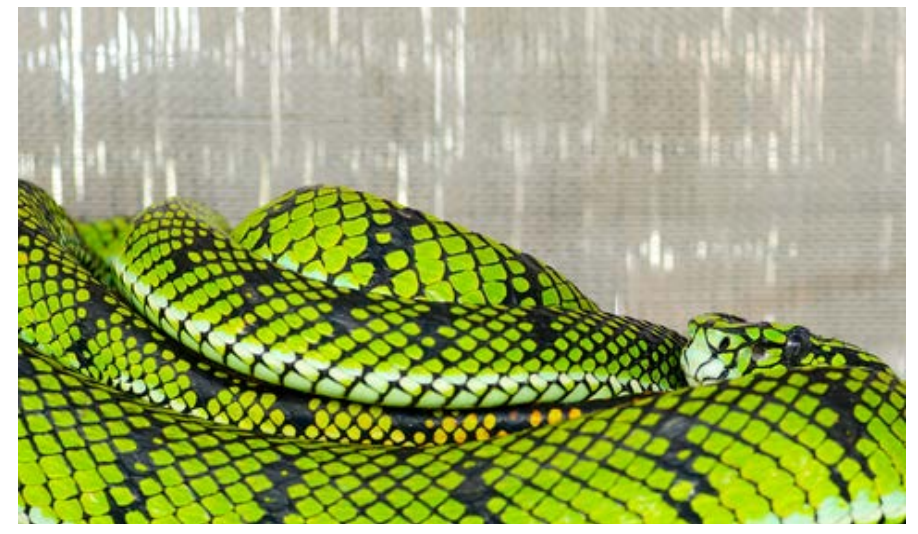

Figure 10. The Sumatran pit viper (Trimeresurus sumatranus) is known to grow over a meter in length, making it one of the larger pit viper species in Malaysia.

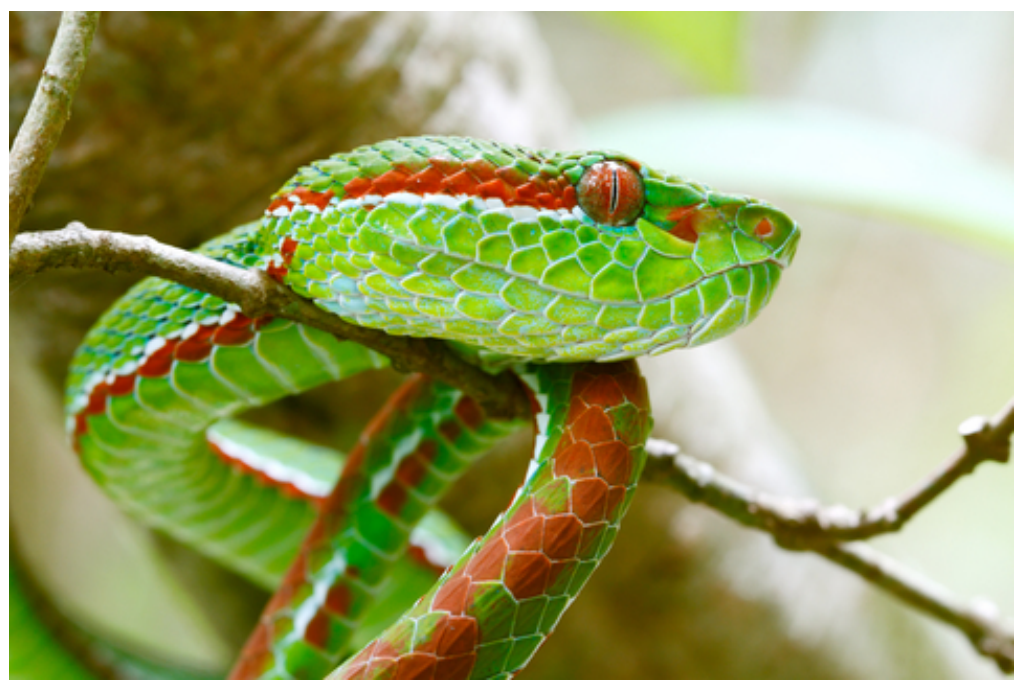

Figure 11. The beautiful pattern on a Pope's tree viper (Trimeresurus popeorum) makes it a really distinctive specimen. As its name implies, it does spend a lot of its time being an arboreal species. 


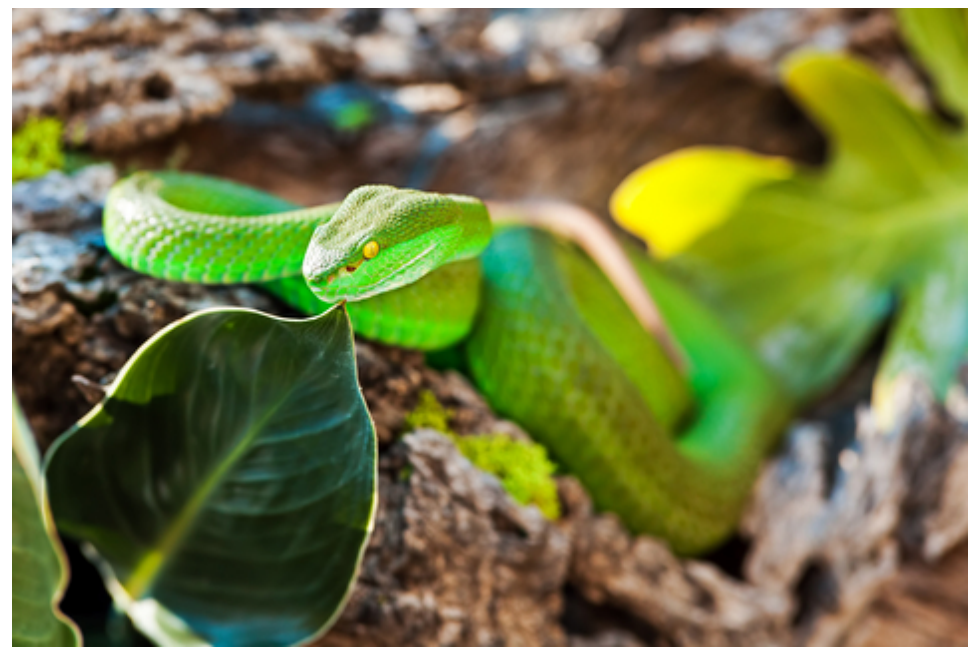

Figure 12. The white lipped pit viper is another species of the pit viper found in Malaysia.

white, though they can come in other colours such as pale green or yellow [7]. The body is green in colour.

\section{First-Aid Treatment of Snake Envenomation [7] [8] [9]}

1) If the snake is killed, it should be brought along with utmost care to the hospital for identification purpose. However, it should always be noted that inappropriate handling may still lead to envenomation despite the snake being dead.

2) It is extremely important to keep the victim calm, without any elements of panic. Reassurance is crucial especially if the victim is anxious. Sometimes, even bites from venomous species may not result in envenomation. This is known as a "dry bite" where a venomous snake bites, but no injection of venom has occurred. Thus, it should be treated as a puncture wound, with consideration to reduce inflammation and subsequent potential infection.

3) It is also important to immobilize the bitten limb with a sling or splint as contraction of muscles will only hasten the spread and absorption of the venom via lymphatics and blood stream.

4) For certain species of snakes (especially elapids such as king cobras, sea snakes), pressure immobilization can be considered using compression bandage that is stretchable and elastic. The bandage is firmly bound starting from distally (toes and fingers) moving up proximally, creating a rigid splint. Ideally, it should not be too tight as to not allow a finger to be slipped between the bandages. However, it is extremely important to take note that pressure immobilization should not be applied in cases of pit viper envenomation as the venom may cause severe local tissue necrosis with increased intra-compartmental pressure.

5) The wound site should not be disturbed so as to reduce local bleeding, venom absorption and to minimize the risk of introduction of infection. Thus, incisions and sucking of the bite wound to "suck out" the venom is highly not recommended. Within one or two minutes of envenomation, the venom would 
have been completely absorbed into the body. The incisions or sucking the wound would only create a portal of entry for secondary infections and as well as aggravate the bleeding.

6) An arterial tourniquet or a tight ligature of any kind is highly not recommended as this can result in limb ischemia. In addition, should the tourniquet be released, venom dissemination would be dramatically increased following the normal physiological response of reactive hyperemia.

\section{Diagnosis: Species Identification}

It is of utmost importance to identify the species of snake that is responsible for the envenomation. This is to ensure effective subsequent management using the proper anti-venom. There are several ways, often used in conjunction when it comes to species identification.

If the snake has been killed and is brought to the hospital, identification can thus be made without any hassle. Otherwise, a history pertaining the bite should be obtained from the patient, focusing on the patient's description on the snake's appearance, such as size, color and shape of the head [9]. It is also crucial to determine how long ago the bite had occurred. This may correlate with the current presentation of the patient at the hospital [9]. Finally, clinical observation and physical examination may reveal certain tell-tale signs that are pathognomonic of certain family of venomous snakes. Systemic viperids envenomation will almost always result in increased bleeding diathesis, accompanied by localized swelling and hemorrhage [7]. Elapids envenomation on the other hand usually results in clinical neurotoxicity, with drowsiness, ptosis, bulbar palsy cranial nerve palsy, flaccid paralysis and finally, respiratory failure [7] [9]. Cobra bites are also associated with local tissue necrosis. Bites from sea snakes are myotoxic and thus, there would be profound myalgia and rhabdomyolysis. Generalized muscle aches and tenderness are also common. The patient's urine may be brownish in color due to myoglobinuria following rhabdomyolysis [9].

An important thing to note is that, there are also numerous non-venomous species in Malaysia. The bites from these species will thus, not result in envenomation and should be managed as a normal puncture wound [9].

\section{Physical Examination}

On inspection of the bitten area, fang marks may be evident, together with swelling. Pain and tenderness upon palpation may be noted. However, some species of sea snakes may result in a virtually painless bite, thus often leading to a delayed management [10]. Regional lymph nodes should also be inspected and palpated. The absence of a pulse, together with cold and immobile limb may suggest compartment syndrome or more rarely, intravascular thrombosis. Persistent bleeding from the bite site may be overt in cases of viperids envenomation [10]. Local tissue necrosis may be evident, demonstrated by darkening of the surrounding skin and loss of sensation at the area affected. This is especially 
common in cases of cobras and pit vipers envenomation with the latter associated with more bleeding and oedema [7].

Vital signs should always be monitored closely. Signs of hypovolemic shock should always be considered especially if there is overt bleeding which is more common in viper species [9]. The gingival sulci should be inspected as these are one of the earliest bleeding sites indicating spontaneous systemic hemorrhage [7]. Besides that, petechiae, purpura and ecchymoses should also be noted. Other hemostatic disturbances that may arise include bleeding from the eyes, epistaxis, hemoptysis and hematemesis [7].

Systemic neurotoxicity can manifest in the body in several ways. Following bites from elapids, there usually would be a flaccid descending paralysis. Upon examination of the eyes, there may be ptosis which can be elicited by asking the patient to look up and see if the eyelids retract fully. Testing for eye movements may reveal ophthalmoplegia. Besides that, signs of bulbar palsy such as dysphagia, dysphonia and the inability to protrude ones tongue can also be pronounced in cases of neurotoxic envenoming. A very distinct finding known as "broken-neck sign" may be produced following paralysis of the neck flexor muscles. Ultimately, diaphragmatic paralysis and paralysis of the intercostal muscles will lead to respiratory failure which can occur as quickly as 30 minutes after being bitten [7].

Following sea snake envenomation, there would be generalized muscle stiffness and tenderness owing to the fact that their venom are highly myotoxic [7] [9]. The breakdown of skeletal muscles (rhabdomyolysis) results in myoglobinemia and myoglobinuria, with the latter causing a brown discoloration of the urine. Acute renal failure may also set in following rhabdomyolysis [7]. Figure 13 shows the case of long term sequelae following envenomation of the shore pit viper (Trimeresurus purpureomaculatus).

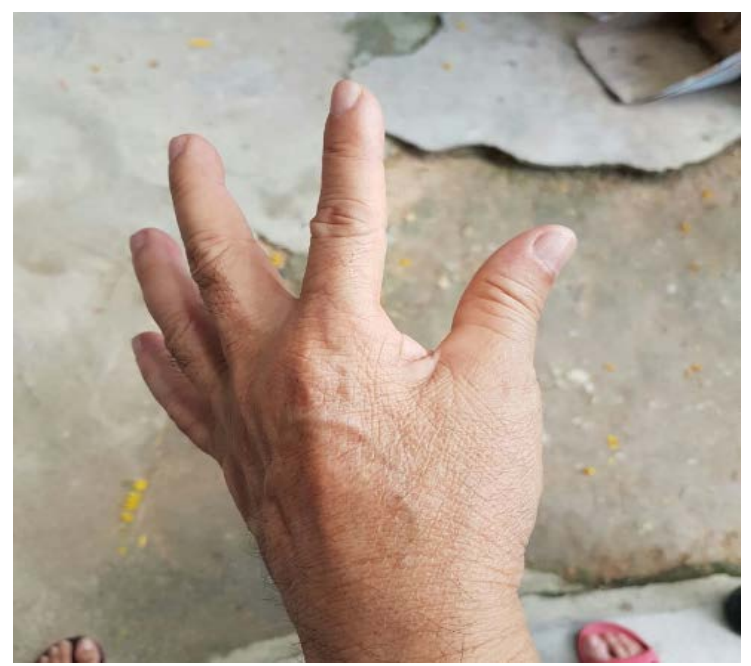

Figure 13. Long term sequelae following envenomation of the shore pit viper (Trimeresurus purpureomaculatus). There is an adduction contracture of the thumb as a result of compartment syndrome and ischemia of the hand due to delayed anti-venom commencement. 


\section{Anti-Venom: The Definitive Treatment}

This targeted and specific therapy is exceedingly effective in reversing the systemic effects of cases of snake envenomation. However, routine administration is not advised as these anti-venoms carry a risk of anaphylaxis [15].

Generally there are 2 types of anti-venoms; Monovalent and Polyvalent anti-venoms. The mono-specific (monovalent) anti-venoms are considered to be more effective in counteracting the toxic effects of venomous snake bites. Besides that, its mono-specific constituents reduce the likelihood of hypersensitivity reactions occurring in recipients. Some examples include anti-Malayan pit viper, anti-Malayan cobra anti-venom and monovalent Thai cobra anti-venom which is effective against both Naja kaouthia and Ophiophagus hannah [7] [15].

Polyvalent anti-venoms are more useful when the species of snake that has bitten the patient is unknown [15]. Such anti-venoms such as the neuro-polyvalent snake anti-venom covers for a wider species such as venoms of Ophiophagus hannah, Naja kaouthia, Bungarus fasciatus and Bungarus candidus. The haemato-polyvalent snake anti-venom is also effective against Calloselasma rhodostoma and other Trimerusurus sp. while exhibiting an extensive cross-neutralization activity against many viperids species [10].

With elapid envenomation, it is wise to anticipate respiratory failure and thus, mechanical ventilation may be inevitable in some severe cases, as diaphragmatic paralysis sets in due to the potent neurotoxicity [15].

While the elapids and sea snakes have a lot in common in terms of toxin components, the viperids family altogether exhibit more signs of haematotoxicity. Prolonged prothrombin time and abnormal INR values are characteristic of viper envenomation. Bleeding tendencies either local or systemic may be fatal and thus, whole blood, or fresh frozen plasma or platelets should be transfused to rectify the bleeding tendency [15]. Occasionally, compartment syndrome may set in following pit viper envenomation, in which case an urgent fasciotomy can be lifesaving. Surgical debridement and desloughing of necrotic areas are also necessary in this setting to ensure proper wound healing [15].

\subsection{Allergic Reactions to Anti-Venom}

Despite being regarded as the gold standard treatment, antivenom may also pose a threat to a handful of patients (approximately $10 \%$ of patients) by triggering an anaphylactic reaction of the body. There are a few clinically important conditions that need to be addressed. Should the treating clinician suspect anti-venom hypersensitivity, the anti-venom should be discontinued immediately [7] [9] [15].

An early hypersensitivity reaction typically commences within minutes of initiation of treatment. Manifestations typical to anaphylaxis will then ensue. These include urticaria, nausea, vomiting, tachycardia and abdominal colic. Life threatening conditions may entail following these signs and symptoms, such as angioedema, hypotension and bronchospasm which ultimately leads to anaphy- 
lactic shock. Approximately $40 \%$ of early hypersensitivity reactions will develop severe systemic anaphylaxis [7] [15]. Epinephrine is the mainstay of treatment, usually given intramuscularly at a dose of 500 microgram for adults or 10 $\mathrm{mcg} / \mathrm{kg}$ for children. Following intramuscular epinephrine, treatment with $\mathrm{H} 1$ receptor antagonist (anti-histamine) is indicated, such as chlorpheniramine. Intravenous hydrocortisone is then given at a dose of $100 \mathrm{mg}$ for adults and 2 $\mathrm{mg} / \mathrm{kg}$ for children. Hypotension is addressed by replacing the intravascular compartment as per emergency shock protocols [9].

Besides the early anaphylactic reaction, a pyrogenic (endotoxin) reactions can occur within 1 or 2 hours of receiving the anti-venom. Patients may be febrile, hypotensive and exhibit chills and rigors. Children may suffer from febrile fits. In addition to the above treatment, anti-pyretics are also indicated (Paracetamol) and other means to cool down the patient's core temperature (cooling blankets or tepid sponging) [9].

The late reaction is known as serum sickness which can develop anywhere between day 1 to day 12 of starting the anti-venom. Signs and symptoms include non-specific findings such as nausea, vomiting and diarrhea while other findings such as lymphadenopathy, urticaria, myalgia, mononeuritis multiplex and proteinurea may also be apparent. Encephalopathy is also seen in some rare cases. The treatment for serum sickness is a 5 days course of oral anti-histamine (chlorphenaramine) and if the victim fails to respond to treatment, they should be started on a 5 days course of prednisolone ( $5 \mathrm{mg}$ six hourly) [9].

\subsection{Cobra-Spit Ophthalmia}

When venom is sprayed into the eyes, thorough irrigation with copious amount of water or normal saline should be done as a means of decontamination. An analgesic with mydriatic activity such as epinephrine (e.g. 0.5\% epinephrine drops) may be used to reduce inflammation and pain [9]. Besides that, fluorescein staining or slit lamp examination is crucial to rule out corneal abrasion. The use of antibiotics is also warranted, such as tetracycline or chloramphenicol to prevent endophthalmitis. Some authors suggest the use of diluted anti-venom, however its benefits are not clear and should be avoided [9].

\section{Conclusion}

Snake envenomation is fairly common in Malaysia, with a handful of it being potentially fatal. There are many reasons contributing to this, including the destruction of their natural habitat which ultimately leads them closer to the dense human population of the urban setting. Whatever the reasons are, prompt treatment is required in cases of envenomation starting from basic first aid knowledge, which has shown to be beneficial and reduces morbidity and mortality. Ultimately, definitive management with antivenom therapy may be indicated in selecting cases. However, this should always be done in a closely monitored environment due to the potential hazardous side effects it poses. 


\section{Funding}

No funding was used for this specific work.

\section{Acknowledgements}

The authors would like to thank the Penang Snake Temple where most of the pictures were taken and a lot were studied regarding each local species and their behaviours.

\section{Conflicts of Interest}

The authors have declared that no competing interests exist in the culmination of this body of work.

\section{References}

[1] Magill, A.J., Strickland, G.T., Maguire, J.H., Ryan, E.T. and Solomon T. (2012) Hunter's Tropical Medicine and Emerging Infectious Disease. Elsevier Health Sciences, Amsterdam.

[2] Nelsen, D.R., Nisani, Z., Cooper, A.M., Fox, G.A., Gren, E.C., Corbit, A.G., et al. (2014) Poisons, Toxungens, and Venoms: Redefining and Classifying Toxic Biological Secretions and the Organisms That Employ Them. Biological Reviews, 89, 450-465. https://doi.org/10.1111/brv.12062

[3] Hodgson, E. (2012) Chapter Fourteen-Toxins and Venoms. Progress in Molecular Biology and Translational Science, Vol. 112, Academic Press, Cambridge, 373-415. https://doi.org/10.1016/B978-0-12-415813-9.00014-3

[4] Mebs, D. (2002) Venomous and Poisonous Animals: A Handbook for Biologists, Toxicologists and Toxinologists, Physicians and Pharmacists. MedPharm, Guildford.

[5] Berthé, R.A. (2011) Spitting Behaviour and Fang Morphology of Spitting Cobras. Doctoral Dissertation, Universität Bonn, Bonn.

[6] Das, I., Ahmed, N. and Liat, L.B. (2015) Venomous Terrestrial Snakes of Malaysia: Their Identity and Biology. In: Gopalakrishnakone, P., Faiz, A., Fernando ,R., Gnanathasan, C., Habib, A. and Yang, C.C., Eds., Clinical Toxinology in Asia Pacific and Africa, Vol. 2, Springer, Dordrecht, 53-69.

https://doi.org/10.1007/978-94-007-6386-9 5

[7] Hong, T.N., Hock, T.C. and Kuan, L.P. A Brief Guide to Snake Envenomations in Malaysia and Antivenom Therapy. Venom and Toxin Research Group, Kuala Lumpur.

[8] Kumar, T., Jayaraman, G., Lee, C.S., Arunkumar, A., Sivaraman, T., Samuel, D., et al. (1997) Snake Venom Cardiotoxins-Structure, Dynamics, Function and Folding. Journal of Biomolecular Structure and Dynamics, 15, 431-463. https://doi.org/10.1080/07391102.1997.10508957

[9] Warrell, D.A. (1999) The Clinical Management of Snake Bites in the Southeast Asian Region. The Southeast Asian Journal of Tropical Medicine and Public Health, 30, 1-67.

[10] Tan, C.H., Tan, K.Y., Fung, S.Y. and Tan, N.H. (2015) Venom-Gland Transcriptome and Venom Proteome of the Malaysian King Cobra (Ophiophagus hannah). BMC Genomics, 16, Article No. 687. https://doi.org/10.1186/s12864-015-1828-2 
[11] Munawar, A., Trusch, M., Georgieva, D., Hildebrand, D., Kwiatkowski, M., Behnken, H., et al. (2014) Elapid Snake Venom Analyses Show the Specificity of the Peptide Composition at the Level of Genera Naja and Notechis. Toxins, 6, 850-868. https://doi.org/10.3390/toxins6030850

[12] Ng, P.K. and Yang, C. (1989) On Michael Willmer Forbes Tweedie. Raffles Bulletin of Zoology, 37, 160-167.

[13] Daltry, J.C., Ponnudurai, G., Shin, C.K., Tan, N.-H., Thorpe, R.S. and Wolfgang, W. (1996) Electrophoretic Profiles and Biological Activities: Intraspecific Variation in the Venom of the Malayan Pit Viper (Calloselasma rhodostoma). Toxicon, 34, 67-79. https://doi.org/10.1016/0041-0101(95)00122-0

[14] Tan, C.H. and Tan, N.H. (2015) Toxinology of Snake Venoms: The Malaysian Context. Snake Venoms, 1-37.

[15] Soo, H.-H., Lau, L.-G. and Chew, P.-H. (2005) Sarawak Handbook of Medical Emergencies. CE Publishing, Quezon City. 